\title{
Effect of Production System (Intensive and Free-Range), Slaughter Age and Gender on Nutrient and Fatty Acid Composition of Meat in Partridges (Alectoris chukar)
}

\author{
Mehmet Akif Boz ${ }^{1, a, *}$, Fatih $\ddot{O} z^{2, b}$, Musa Sarıca ${ }^{3, c}$, Umut Sami Yamak ${ }^{3, d}$ \\ ${ }^{1}$ Department of Animal Science, Faculty of Agriculture, Yozgat Bozok University, 66900 Yozgat, Turkey \\ ${ }^{2}$ Department of Food Engineering, Faculty of Agriculture, Atatürk University, 25240, Erzurum, Turkey \\ ${ }^{3}$ Department of Animal Science, Faculty of Agriculture, Ondokuz Mayis University, 55139, Samsun, Turkey
} *Corresponding author

\section{A R T I C L E I N F O \\ ${ }^{\#}$ This study was supported by Yozgat Bozok University (Project number: 6602b-ZF/16-15). \\ Research Article}

Received : 27/11/2020

Accepted : 02/03/2021

Keywords:

Partridges

Fatty acid

Production systems

Age

Gender

\section{A B S T R A C T}

This study was conducted to determine the effect of production system, slaughter age, and gender on the nutrient composition, fatty acids profile, and index values of breast and thigh meat in Alectoris chukar partridges. Partridges were slaughtered at 14, 16, and 18 weeks of age and skinless breast and thigh meat of male and female were used in the study (a total of 96 samples in 2 replicates). The production system affected only the crude fat level of the thigh meat in terms of nutrient composition and it was found higher in the intensive system compared to the free-range system. Age and gender did not significantly affect the composition of breast and thigh meat. In terms of fatty acid profile, erucic acid (C22:1n9) level in breast meat was higher in intensive system, while it was higher in thigh meat in free-range. While the percentage of docosahexaenoic acid (C22:6n3) increased with age in breast meat, it decreased in thigh meat with age. While eicosenoic acid (C20:1) percentage was higher in breast meat of male birds compared to females, only stearic acid (C18:0) was found to be higher in thigh meat. Saturated fatty acids (SFA), linolenic acid (n3), thrombogenic index (TI) and atherogenic index (AI) values were higher in thigh meat produced in free-range system, while polyunsaturated fatty acids (PUFA), total unsaturated fatty acids (UFA), linoleic acid (n6) and hypocholesterolaemic / hypercholesterolaemic ratio $(\mathrm{h} / \mathrm{H})$ were significantly higher in intensive system. While SFA and TI levels in thigh meat decreased with age, monounsaturated fatty acids (MUFA), UFA and oleic acid (n9) percentages increased. MUFA / SFA in thigh meat of females was higher than males, other indexes were found insignificant. The results shows that partridges had desirable fatty acid composition. Especially, the increase in MUFA and UFA values with age in thigh meat compared to breast indicates that thigh meat is enriched in terms of unsaturated fatty acids. However, the higher SFA and AI values obtained in the free-range system could be considered a negative outcome for alternative production systems that prioritize bird welfare and consumer demands.

\section{Introduction}

In recent years, there has been an increase in the consumption of alternative poultry meat such as quail, pheasant, partridge, guinea fowl and ostriches, and the production of these species is increasing day by day (Alva et al., 2015; Yamak et al., 2018; Sarica et al., 2019). Nowadays, partridges and pheasants are the leading game animals produced by humans for hunting and consumption and they are grown in various parts of the world (Yamak, 2015). They are released into hunting areas for tourism and have a significant return on investment. However, meatbased production has also become more common and the studies conducted on partridge demonstrate that these birds could be raised for meat production (Yamak et al., 2016).
In a study conducted on partridges, it was determined that the production system, slaughter age and gender had a significant effect on body weight, carcass weight, breast and thigh weight (Yamak et al., 2016). It has been observed that 18-week-old male partridges reared in closed system have higher yield values. In the same study, breast and thigh meat weights were determined as 141.6, 148.5, 155.3 $\mathrm{g}$ and 94.04, 98.03, 100.1 $\mathrm{g}$ at 14, 16 and 18 weeks of age, respectively. Cold carcass percentage varies between $76.4 \%$ and $77.9 \%$. Breast and thigh percentage according to cold carcass varies between $36.5 \%-37.7 \%$ and $23.9 \%$ $24.7 \%$, respectively (Yamak et al., 2016). Partridge meat has a high nutritional value (approximately, $240 \mathrm{~g}$ crude 
protein/kg and $603 \mathrm{~kJ} / 100 \mathrm{~g}$ meat). Although partridge is generally known as a game bird, it has recently increased in popularity in the gourmet market (Ozek et al., 2003; Vitula et al., 2011; Yamak et al., 2016).

Genotype and production system are seen as the most important factors affecting meat quality in poultry. The effect of free-range system on meat quality depends on slaughter age, physical activity, and its ability to reach feed stuffs in the outdoor environment (BogosavljevicBoskovic et al., 2012). Production system is a very important parameter among non-genetic factors affecting slaughter, carcass and meat quality traits (Meluzzi et al., 2009). However, gender and environmental conditions (management, nutrition, slaughter, etc.) are also important factors that affect poultry meat quality. The flavor of meat reaches its peak with sexual maturation, and earlier slaughter age changes the flavor. Moreover, the meat is generally softer and tender when the birds are slaughtered at early ages (Berri, 2000). In general, body and muscle composition change with age since protein, fat, and dry matter percentages increase over time (Aberle et al., 2001; Yamak et al., 2020).

In this study, the effects of production system, slaughter age and gender on nutrient composition and fatty acid profile of meat in partridges (Alectoris chukar) were determined.

\section{Materials and Methods}

A total of 400-day-old partridge chicks were placed in the intensive and free-range systems, where they were randomly allocated to pens as follows: four pens per system and 50 chicks per pen. Then, the pens were interspersed with windowed houses. The pen sizes were $3.5 \times 3.5 \mathrm{~m}$ and covered with $0.5 \times 0.5 \mathrm{~cm}$ wire mesh, which separated the pens to prevent birds from flying to the other pens. The stocking density was provided as 4 birds $/ \mathrm{m}^{2}$ in intensive system and $1 \mathrm{bird} / \mathrm{m}^{2}$ in free-range system. One round feeder and round drinker were located in each pen. Wood shavings ( $8 \mathrm{~cm}$ thick) were used as litter in the indoor pens, and no fresh litter was added during the experiment. The necessary temperature was achieved using infra-red heaters, and the lighting were provided by incandescent bulbs. The lighting was 24 $\mathrm{h}$ /day during the first three days. The chicks received light for $20 \mathrm{~h} /$ day for 6 weeks. From 6 weeks of age to slaughter, natural lighting was used for $14 \mathrm{~h} /$ day. The birds in the free-range system were provided with $24 \mathrm{~h}$ access to outdoor pens $(14 \times 3.5 \mathrm{~m})$ through a single doorway $(50 \times 90 \mathrm{~cm})$ after 6 weeks of age. Access to the outdoor area was arranged after the 6 weeks of age, depending on seasonal conditions. The average temperature in the free-range (outdoor area) was $29^{\circ} \mathrm{C}(26$ to $31^{\circ} \mathrm{C}$ ) and humidity $64 \%(52$ to $76 \%)$, while the average temperature inside was $25^{\circ} \mathrm{C}\left(23\right.$ to $\left.29^{\circ} \mathrm{C}\right)$ and humidity $62 \%$ (51 to $76 \%$ ) throughout the study. The natural ventilation was acquired with windows, and artificial ventilation sometimes was carried out with 2 additional fans when needed. All birds were fed ad libitum with broiler starter diet (commercial, maize and soybean meal based) containing $230 \mathrm{~g}$ crude protein $/ \mathrm{kg}$ and 12.8 MJ ME, $13.5 \mathrm{~g}$ lysine, $4.50 \mathrm{~g}$ methionine, 10.0 g Ca, 5.00 g P, 120 mg Mn, 15 mg Cu, 100 mg Zn, 12000
IU Vitamin A, and 4200 IU Vitamin D per kg (estimated values). There were Medicago sativa L. and Lolium spp. between the 6-9 weeks of the study in the outdoor area. In this 3-week period, the vegetation in the outdoor area was consumed ad-libitum. After this week, there was no vegetation for consumption.

Water was also given ad libitum. 5 male and 5 female partridges from each pen with body weight values closest to the average body weight were selected and were slaughtered at 14,16 and 18 weeks of age. The gender was determined before slaughter based on the calcar and the biological structure; which was confirmed after slaughter. Analyzes were performed on a total of 96 samples in 2 replicates from a total of 48 breast and meat samples of partridge. The body weight of these birds was recorded after 8-h fasting period and before they were slaughtered. Semi-automated equipment was used for the procedures of scalding $\left(1 \mathrm{~min}\right.$. at $\left.56^{\circ} \mathrm{C}\right)$, plucking, coldwater chilling, vent opening, evisceration, and airchilling. Carcasses were cut into parts according to standard methods (Sarica et al., 2011).

The meat samples were stored within a temperature range of -18 to $-22^{\circ} \mathrm{C}$ immediately following slaughter. The analyses were conducted on 48 pectorales major and minor of breast and the whole muscle of both thighs.

The chemical composition of meat was analyzed on skinless breast and thigh meat stored at $-18^{\circ} \mathrm{C}$. Dry matter, crude protein, crude fat, and ash contents were determined according to the methods described by Gokalp et al. (2010). The AOAC 996.01 method was used to determine the composition of fatty acid methyl esters (Satchithanandam et al., 2001). A total of $0.1 \mathrm{~g}$ fat obtained by the ether extraction method was shaken with $10 \mathrm{~mL} \mathrm{n-}$ hexane and $0.5 \mathrm{~mL} 2 \mathrm{~N}$ methanolic potassium hydroxide solution, and the mixture was shaken again, kept in the dark for $1-2 \mathrm{~h}$, and $1 \mu \mathrm{L}$ from the upper phase was directly injected into the gas chromatograph with a flame ionization detector. The fatty acid composition was analysed using a Shimadzu gas chromatograph (Model QP2010 Plus) with a Restek RTX-2330 capillary column (60 m, 0.25 mm i.d., $0.1 \mu \mathrm{m}$ film thickness, Bellefonte, PA, USA) and a flame ionization detector (FID). After the column oven temperature was maintained at $100^{\circ} \mathrm{C}$ for $3 \mathrm{~min}$, it was increased by $4^{\circ} \mathrm{C}$ per min until it reaches $240^{\circ} \mathrm{C}$, which was maintained for $18 \mathrm{~min}$. The injection temperature was $250^{\circ} \mathrm{C}$ and the detector temperature was $255^{\circ} \mathrm{C}$. Helium was used as the carrier gas, and the flow rate was 0.64 $\mathrm{mL} / \mathrm{min}$. The injection split ratio was $1: 80$. The LabSolution computer program was used to control the GC/FID system, and the standard 37 fatty acid methyl ester (FAME) mix (Supelco) was used. FAME peaks were identified by comparing the results against the retention times and chain lengths specified by the FAME standard. The international abbreviations of the fatty acids used in the present study are as follows; Myristic acid (C14:0), palmitic acid (C16:0), stearic acid (C18:0), oleic acid (C18:1n9c), linoleic acid (C18:2n6c), linolenic acid (C18:3n3), eicosenoic acid (C20:1), behenic acid (C22:0), erucic acid (C22:1n9), docosahexaenoic acid (C22:6n3 or DHA), saturated fatty acids (SFA), monounsaturated fatty acids (MUFA), polyunsaturated fatty acids (PUFA), total unsaturated fatty acids (UFA). 
In the present study, the atherogenic index (AI) and thrombogenic index (TI) were determined according to the method of Peiretti and Meineri (2008), the nutritive value (NV) and desirable fatty acids (DFA) indexes were determined according to the method of Caneque et al. (2005), and the hypocholesterolaemic/hypercholesterolaemic ratio $(\mathrm{h} / \mathrm{H})$ was calculated according to the formulas of Ahmed et al. (2015).

$$
\begin{gathered}
\mathrm{AI}=(\mathrm{C} 12: 0+(4 \times \mathrm{C} 14: 0)+\mathrm{C} 16: 0) / \sum \mathrm{UFA} \\
\mathrm{TI}=(\mathrm{C} 14: 0+\mathrm{C} 16: 0+\mathrm{C} 18: 0) /\left[(0.5 \times \mathrm{MUFA})+\left(0.5 \times \sum \mathrm{n} 6\right)\right. \\
\left.+\left(3 \times \sum \mathrm{n} 3\right)+\left(\sum \mathrm{n} 3 / \sum \mathrm{n} 6\right)\right] ; \\
\mathrm{NV}=(\mathrm{C} 18: 0+\mathrm{C} 18: 1) / \mathrm{C} 16: 0 ; \\
\mathrm{DFA}=(\mathrm{C} 18: 0+\mathrm{UFA}) ;
\end{gathered}
$$

$\mathrm{h} / \mathrm{H}=[($ sum of $\mathrm{C} 18: 1$ cis-9, C18:2 n6, C20:4 n6, C18:3 n3, C20:3 n6, C20:5 n3, and C22:6 n3) / (sum of C14:0 and

$$
\text { C16:0)]. }
$$

Undetected and trace amounts of fatty acids were not provided in the tables. The total and index values were calculated based on all fatty acids.

\section{Statistical Analysis}

Analysis of variance (ANOVA- Bootstrap Analysis) with a factorial arrangement (production system, age and gender) was used to test the effects of production system (intensive and free range), slaughter age (14, 16 and 18 weeks of age) and the 2 or 3-Way interactions between production system, slaughter age and gender. The Duncan test was performed for multiple comparisons of means. A level of $\mathrm{P}<0.05$ was considered statistically significant. Statistical analysis was performed using the SPSS Version 20 (Yamak et al., 2016).

\section{Results}

Nutrient composition of breast and thigh meat of partridges are given in Table 1 . The crude fat content in thigh meat of partridges reared in the intensive system was significantly higher than free-range system $(\mathrm{P}<0.05$, Table $1)$. Age $\times$ Gender interaction on dry matter percentage in breast meat was found significant $(\mathrm{P}<0.05)$.

Fatty acid profiles of breast and thigh meat in partridges are shown in Table 2 and Table 3. Oleic (C18:1n9c), linoleic (C18:2n6c), and palmitic (C16:0) acids comprised the greatest percentage $(<20.0 \%)$ of fatty acids in both thigh and breast meat.

Table 1. Nutrient composition of breast and thigh meat in partridges $(\%)$

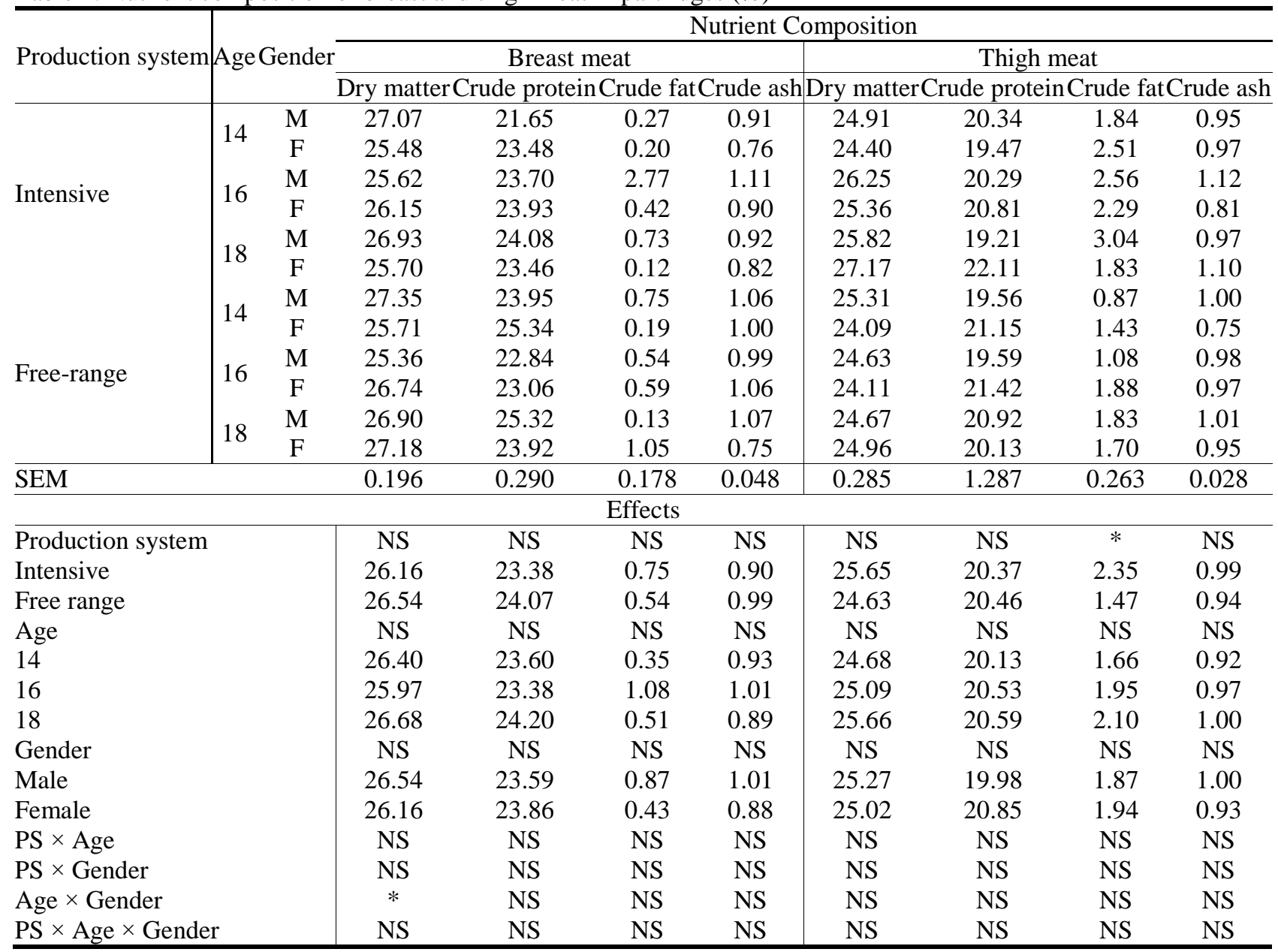

M: male, F: female, PS: production system, SEM: standard error of the mean, NS: P $>0.05, * \mathrm{P}<0.05$. 
Table 2. Fatty acid profile of breast meat in partridges $(\%)$

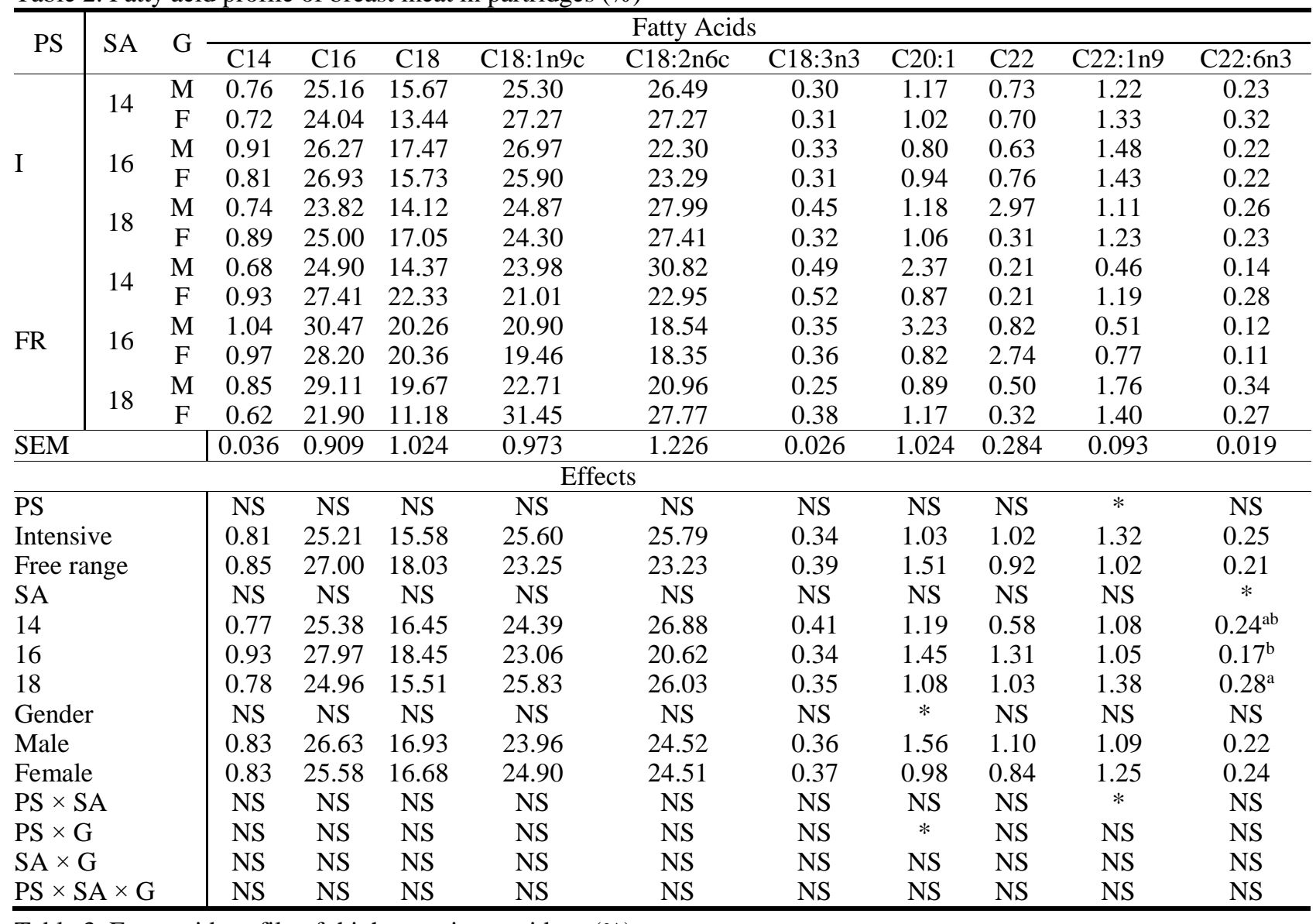

Table 3. Fatty acid profile of thigh meat in partridges (\%)

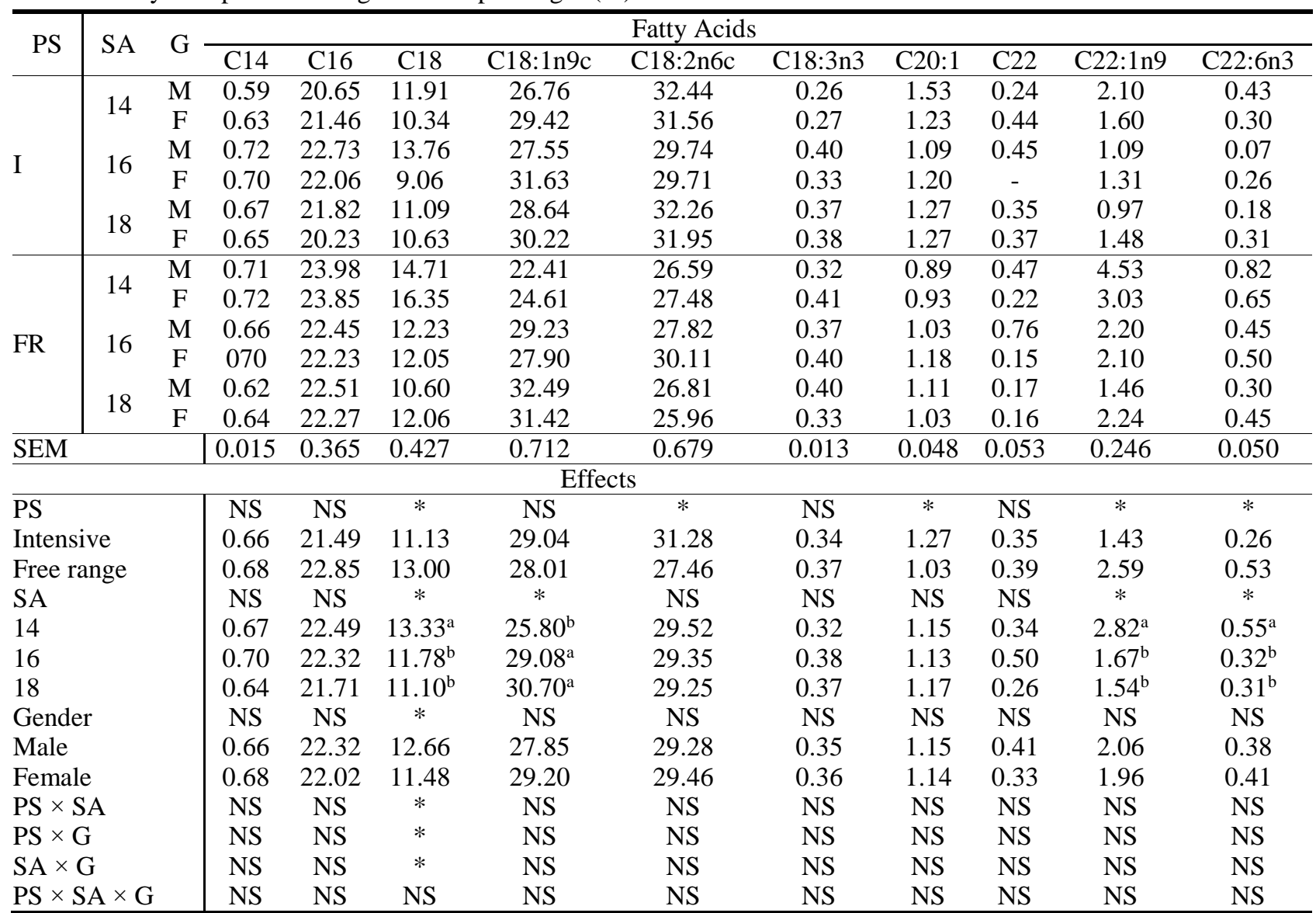


Table 4. Fatty acid balance of breast meat in partridges (\%)

\begin{tabular}{|c|c|c|c|c|c|c|c|c|c|}
\hline \multirow{2}{*}{ PS } & \multirow{2}{*}{ Age } & \multirow{2}{*}{ G } & \multicolumn{7}{|c|}{ Fatty acids } \\
\hline & & & SFA & MUFA & PUFA & UFA & $n-3$ & $n-6$ & $n-9$ \\
\hline \multirow{6}{*}{ I } & \multirow{2}{*}{14} & $\mathrm{M}$ & 43.32 & 29.30 & 27.37 & 56.68 & 0.59 & 26.49 & 26.62 \\
\hline & & $\mathrm{F}$ & 39.46 & 31.90 & 28.64 & 60.54 & 1.09 & 27.27 & 28.77 \\
\hline & \multirow{2}{*}{16} & M & 46.63 & 30.08 & 23.28 & 55.46 & 0.62 & 22.33 & 27.50 \\
\hline & & $\mathrm{F}$ & 44.53 & 31.01 & 24.45 & 53.36 & 0.61 & 23.44 & 27.39 \\
\hline & \multirow{2}{*}{18} & $\mathrm{M}$ & 42.22 & 28.46 & 29.31 & 57.77 & 0.72 & 28.22 & 25.98 \\
\hline & & $\mathrm{F}$ & 43.99 & 27.79 & 28.21 & 56.00 & 0.61 & 27.41 & 25.54 \\
\hline \multirow{6}{*}{ FR } & \multirow{2}{*}{14} & M & 40.73 & 26.40 & 32.87 & 59.27 & 0.73 & 31.54 & 24.44 \\
\hline & & $\mathrm{F}$ & 51.83 & 24.00 & 24.17 & 48.17 & 0.89 & 22.95 & 22.20 \\
\hline & \multirow{2}{*}{16} & M & 53.53 & 26.54 & 19.92 & 46.46 & 0.68 & 18.78 & 21.46 \\
\hline & & $\mathrm{F}$ & 55.07 & 22.69 & 22.23 & 44.93 & 0.78 & 20.95 & 20.24 \\
\hline & \multirow{2}{*}{18} & M & 50.83 & 27.06 & 22.10 & 49.17 & 0.69 & 20.96 & 24.47 \\
\hline & & $\mathrm{F}$ & 34.53 & 36.68 & 28.78 & 65.47 & 0.74 & 27.77 & 32.93 \\
\hline \multicolumn{3}{|l|}{ SEM } & 1.981 & 1.015 & 1.188 & 1.981 & 0.041 & 1.212 & 0.993 \\
\hline \multicolumn{10}{|c|}{ Effects } \\
\hline \multicolumn{3}{|l|}{ PS } & NS & NS & NS & NS & NS & NS & NS \\
\hline \multicolumn{3}{|l|}{ I } & 43.36 & 29.76 & 26.88 & 56.64 & 0.71 & 25.86 & 26.97 \\
\hline \multicolumn{3}{|l|}{ FR } & 47.75 & 27.23 & 25.01 & 52.25 & 0.75 & 23.83 & 24.29 \\
\hline \multicolumn{3}{|l|}{ Age } & NS & NS & NS & NS & NS & NS & NS \\
\hline \multicolumn{3}{|l|}{14} & 43.83 & 27.90 & 28.26 & 56.16 & 0.83 & 27.06 & 25.51 \\
\hline \multicolumn{3}{|l|}{16} & 49.94 & 27.58 & 22.47 & 50.06 & 0.67 & 21.38 & 24.15 \\
\hline \multicolumn{3}{|l|}{18} & 42.89 & 30.00 & 27.10 & 57.10 & 0.69 & 26.09 & 27.23 \\
\hline \multicolumn{3}{|l|}{ Gender } & NS & NS & NS & NS & NS & NS & NS \\
\hline \multicolumn{3}{|l|}{ M } & 46.21 & 27.98 & 25.81 & 53.79 & 0.67 & 24.72 & 25.08 \\
\hline \multicolumn{3}{|l|}{$\mathrm{F}$} & 44.90 & 29.01 & 26.08 & 55.10 & 0.79 & 24.97 & 26.18 \\
\hline \multicolumn{3}{|l|}{$\mathrm{PS} \times \mathrm{SA}$} & NS & NS & NS & NS & NS & NS & NS \\
\hline \multicolumn{3}{|l|}{$\mathrm{PS} \times \mathrm{G}$} & NS & NS & NS & NS & NS & NS & NS \\
\hline \multicolumn{3}{|l|}{$\mathrm{SA} \times \mathrm{G}$} & NS & NS & NS & NS & NS & NS & NS \\
\hline \multicolumn{3}{|c|}{$\mathrm{PS} \times \mathrm{SA} \times \mathrm{G}$} & NS & NS & NS & NS & NS & NS & NS \\
\hline
\end{tabular}

Table 5. Fatty acid balance of thigh meat in partridges $(\%)$

\begin{tabular}{|c|c|c|c|c|c|c|c|c|c|}
\hline \multirow{2}{*}{ PS } & \multirow{2}{*}{ Age } & \multirow{2}{*}{$\mathrm{G}$} & \multicolumn{7}{|c|}{ Fatty acids } \\
\hline & & & SFA & MUFA & PUFA & UFA & $n-3$ & $n-6$ & $n-9$ \\
\hline \multirow{6}{*}{ I } & \multirow{2}{*}{14} & $\mathrm{M}$ & 34.10 & 32.21 & 33.68 & 65.89 & 0.87 & 32.44 & 28.86 \\
\hline & & $\mathrm{F}$ & 33.12 & 34.19 & 32.68 & 66.87 & 0.75 & 31.65 & 31.02 \\
\hline & \multirow{2}{*}{16} & $\mathrm{M}$ & 38.37 & 31.09 & 30.53 & 61.62 & 0.64 & 29.74 & 28.66 \\
\hline & & $\mathrm{F}$ & 32.34 & 26.87 & 30.78 & 67.65 & 0.77 & 29.71 & 32.94 \\
\hline & \multirow{2}{*}{18} & M & 34.27 & 32.48 & 33.24 & 65.72 & 0.67 & 32.26 & 29.62 \\
\hline & & $\mathrm{F}$ & 32.21 & 34.60 & 33.18 & 67.78 & 0.88 & 31.95 & 31.70 \\
\hline \multirow{6}{*}{ FR } & \multirow{2}{*}{14} & M & 41.80 & 29.35 & 28.85 & 58.20 & 1.65 & 26.59 & 27.01 \\
\hline & & $\mathrm{F}$ & 40.40 & 30.30 & 29.30 & 59.60 & 1.32 & 27.48 & 27.67 \\
\hline & \multirow{2}{*}{16} & M & 36.05 & 34.78 & 29.16 & 63.94 & 0.98 & 27.82 & 31.46 \\
\hline & & $\mathrm{F}$ & 35.34 & 33.11 & 31.54 & 64.65 & 1.10 & 30.11 & 30.02 \\
\hline & \multirow{2}{*}{18} & $\mathrm{M}$ & 34.48 & 37.60 & 27.92 & 65.52 & 0.84 & 26.81 & 33.98 \\
\hline & & $\mathrm{F}$ & 35.71 & 37.04 & 27.25 & 64.29 & 0.97 & 25.96 & 33.69 \\
\hline \multicolumn{3}{|l|}{ SEM } & 0.679 & 0.628 & 0.675 & 0.680 & 0.066 & 0.680 & 0.545 \\
\hline \multicolumn{10}{|c|}{ Effects } \\
\hline \multicolumn{3}{|l|}{$\overline{\mathrm{PS}}$} & $*$ & NS & $*$ & $*$ & $*$ & $*$ & NS \\
\hline \multicolumn{3}{|c|}{ Intensive } & 34.07 & 33.58 & 32.35 & 65.93 & 0.77 & 31.30 & 30.47 \\
\hline \multicolumn{3}{|c|}{ FR } & 37.30 & 33.71 & 29.00 & 62.70 & 1.14 & 27.46 & 30.64 \\
\hline \multicolumn{3}{|l|}{ Age } & $*$ & $*$ & NS & * & $*$ & NS & $*$ \\
\hline \multicolumn{3}{|l|}{14} & $37.36^{\mathrm{a}}$ & $31.51^{\mathrm{b}}$ & 31.13 & $62.64^{\mathrm{b}}$ & $1.15^{\mathrm{a}}$ & 29.54 & $28.64^{\mathrm{b}}$ \\
\hline \multicolumn{3}{|l|}{16} & $35.53^{\mathrm{ab}}$ & $33.97^{\mathrm{a}}$ & 30.50 & $64.47^{\mathrm{ab}}$ & $0.87^{b}$ & 29.35 & $30.77^{\mathrm{ab}}$ \\
\hline \multicolumn{3}{|l|}{18} & $34.17^{\mathrm{b}}$ & $35.43^{\mathrm{a}}$ & 30.40 & $65.83^{\mathrm{a}}$ & $0.84^{\mathrm{b}}$ & 29.25 & $32.25^{\mathrm{a}}$ \\
\hline \multicolumn{3}{|l|}{ Gender } & NS & NS & NS & NS & NS & NS & NS \\
\hline \multicolumn{3}{|l|}{ M } & 36.51 & 32.92 & 30.56 & 63.48 & 0.94 & 29.28 & 29.93 \\
\hline \multicolumn{3}{|l|}{$\mathrm{F}$} & 34.86 & 34.35 & 30.79 & 65.14 & 0.97 & 29.48 & 31.18 \\
\hline \multicolumn{3}{|c|}{$\mathrm{PS} \times \mathrm{SA}$} & $*$ & $*$ & NS & $*$ & NS & NS & $*$ \\
\hline \multicolumn{3}{|c|}{$P S \times G$} & NS & NS & NS & NS & NS & NS & NS \\
\hline \multicolumn{3}{|c|}{$\mathrm{SA} \times \mathrm{G}$} & NS & NS & NS & NS & NS & NS & NS \\
\hline \multicolumn{3}{|c|}{$\mathrm{PS} \times \mathrm{SA} \times \mathrm{G}$} & NS & NS & NS & NS & NS & NS & NS \\
\hline
\end{tabular}

PS: production system G: gender, I: intensive, FR: free range, M: male, F: female, SFA: saturated fatty acids, MUFA: monounsaturated fatty acids, PUFA: polyunsaturated fatty acids, UFA: total unsaturated fatty acids, n3: omega 3,n6: omega 6, n9: omega 9, SEM: standard error of means, NS: not significant, $* \mathrm{P}<0.05$, ${ }^{\mathrm{a}, \mathrm{b}}$ differences in superscript letters within columns represent significant differences among the groups. 
Linoleic acid (C18:2n6c) was identified as the most common fatty acid in breast meat at 14 weeks of age $(\mathrm{P}<0.05)$. Erucic acid $(\mathrm{C} 22: 1 \mathrm{n} 9)$ was higher in breast meat of partridges reared in the intensive system $(\mathrm{P}<0.05)$.

DHA (C22:6n3) was affected by age and it was found at the lowest level at 16 weeks of age. Eicosenoic acid (C20:1) also was affected by gender and found higher in male partridges compared to females $(\mathrm{P}<0.05)$.

Stearic acid (C18:0), erucic acid (C22:1n9), and DHA (C22:6n3) were found higher in the thigh meat of partridges reared under the free-range system, whereas linoleic acid (C18:2n6c) and eicosenoic acid (C20:1) were higher in the intensive system $(\mathrm{P}<0.05)$. Stearic acid (C18:0), erucic acid (C22:1n9), and DHA (C22:6n3) were higher and the oleic acid $(\mathrm{C} 18: 1 \mathrm{n} 9 \mathrm{c})$ percenatge was lower at 14 weeks of age $(\mathrm{P}<0.05)$. $P S \times G$ interaction for eicosenoic acid (C20: 1) and PS $\times$ SA interaction for erucic acid (C22: 1n9) were found significant in breast meat $(\mathrm{P}<0.05)$. $\mathrm{PS} \times \mathrm{SA}, \mathrm{PS} \times \mathrm{G}$ and $\mathrm{SA} \times \mathrm{G}$ interactions were significant for stearic acid $(\mathrm{C} 18: 0)$ in thigh meat $(\mathrm{P}<0.05)$.

Fatty acid balance and index values for breast and thigh meat in partridges are given in Table 4, Table 5, Table 6 and Table 7, respectively. The production system, slaughter age, and gender had no significant effect on the total fatty acid balance and the index values of breast meat (Table 4 and Table 6; $\mathrm{P}>0.05$ ). There were no significant interactions for fatty acid balance in breast meat $(\mathrm{P}>0.05)$.

In thigh meat, the SFA, n-3, AI, and TI were higher in the free-range system, while the PUFA, UFA, n-6,
PUFA/SFA, and $\mathrm{h} / \mathrm{H}$ were higher in thigh meats produced in intensive system (Table 5 and Table $7 ; \mathrm{P}<0.05$ ). Moreover, the SFA, TI, and n-3 were at the highest levels at 14 weeks of age, whereas MUFA, UFA, n-9, and MUFA/SFA were at the highest levels at 18 weeks $(\mathrm{P}<0.05)$. Finally, the MUFA/SFA value was found higher in females compared to males $(\mathrm{P}<0.05)$. $\mathrm{PS} \times \mathrm{SA}$ interaction was found significant for SFA, MUFA, UFA, n-9, MUFA/SFA and TI in thigh meat $(\mathrm{P}<0.05)$. Also, $\mathrm{PS} \times \mathrm{G}$ interaction was found significant for MUFA/SFA $(\mathrm{P}<0.05)$.

\section{Discussion}

The meat quality is determined by major components such as chemical composition, dry matter, protein, fat, and ash content. In the present study, production system, slaughter age, and gender did not significantly affect the nutrient composition of meat in partridges, with the exception of the thigh meat fat percentage (Table 1). However, contrary to our study results, it was reported that genotype, nutrition, production system, season, and age affect the meat quality traits in poultry species (Castellini et al., 2002; Boz et al., 2017).

Moro et al. (2006) determined the dry matter percentage of local partridges change between 55.9 and $62.4 \%$. They also determined that the protein, fat, and ash percentages of partridge meat were $25.2-29.1 \%, 1.6-5.6 \%$, and $1.2-1.4 \%$, respectively.

Table 6. Fatty acid index values of breast meat in partridges

\begin{tabular}{|c|c|c|c|c|c|c|c|c|c|}
\hline \multirow{2}{*}{ PS } & \multirow{2}{*}{ Age } & \multirow{2}{*}{$\mathrm{G}$} & \multicolumn{7}{|c|}{ Fatty acid index } \\
\hline & & & MUFA/SFA & PUFA/SFA & $\mathrm{NV}$ & $\overline{\mathrm{AI}}$ & TI & $\mathrm{h} / \mathrm{H}$ & DFA \\
\hline \multirow{6}{*}{ I } & \multirow{2}{*}{14} & $\mathrm{M}$ & 0.70 & 0.65 & 1.64 & 0.51 & 1.43 & 2.05 & 72.35 \\
\hline & & $\mathrm{F}$ & 0.81 & 0.73 & 1.70 & 0.45 & 1.16 & 2.24 & 73.98 \\
\hline & \multirow{2}{*}{16} & M & 0.66 & 0.51 & 1.67 & 0.57 & 1.62 & 1.83 & 70.83 \\
\hline & & $\mathrm{F}$ & 0.72 & 0.57 & 1.56 & 0.56 & 1.54 & 1.83 & 71.19 \\
\hline & \multirow{2}{*}{18} & M & 0.73 & 0.75 & 1.67 & 0.48 & 1.32 & 2.27 & 73.05 \\
\hline & & $\mathrm{F}$ & 0.70 & 0.71 & 1.70 & 0.55 & 1.58 & 2.15 & 71.90 \\
\hline \multirow{6}{*}{ FR } & \multirow{2}{*}{14} & $\mathrm{M}$ & 0.85 & 1.12 & 1.79 & 0.56 & 1.52 & 2.87 & 73.64 \\
\hline & & $\mathrm{F}$ & 0.46 & 0.47 & 1.58 & 0.65 & 1.93 & 1.57 & 70.50 \\
\hline & \multirow{2}{*}{16} & M & 0.50 & 0.37 & 1.37 & 0.75 & 2.09 & 1.30 & 66.72 \\
\hline & & $\mathrm{F}$ & 0.42 & 0.41 & 1.42 & 0.74 & 2.12 & 1.46 & 65.29 \\
\hline & \multirow{2}{*}{18} & M & 0.55 & 0.45 & 1.47 & 0.68 & 1.97 & 1.51 & 68.84 \\
\hline & & $\mathrm{F}$ & 1.06 & 0.83 & 1.95 & 0.37 & 0.98 & 2.65 & 76.65 \\
\hline \multicolumn{3}{|l|}{ SEM } & 0.056 & 0.068 & 0.060 & 0.039 & 0.120 & 0.157 & 1.041 \\
\hline \multicolumn{10}{|c|}{ Effects } \\
\hline \multicolumn{3}{|c|}{ Production system } & NS & NS & $\mathrm{NS}$ & NS & NS & NS & NS \\
\hline \multicolumn{3}{|c|}{ Intensive } & 0.72 & 0.65 & 1.66 & 0.52 & 1.44 & 2.06 & 72.22 \\
\hline \multicolumn{3}{|c|}{ Free range } & 0.64 & 0.61 & 1.60 & 0.63 & 1.77 & 1.90 & 70.27 \\
\hline \multicolumn{3}{|l|}{ Age } & NS & NS & NS & NS & NS & NS & NS \\
\hline \multicolumn{3}{|l|}{14} & 0.71 & 0.74 & 1.68 & 0.54 & 1.51 & 2.18 & 72.62 \\
\hline \multicolumn{3}{|l|}{16} & 0.57 & 0.47 & 1.50 & 0.65 & 1.84 & 1.61 & 68.51 \\
\hline \multicolumn{3}{|l|}{18} & 0.76 & 0.69 & 1.70 & 0.52 & 1.46 & 2.15 & 72.61 \\
\hline \multicolumn{3}{|l|}{ Gender } & NS & NS & NS & NS & NS & NS & NS \\
\hline \multicolumn{3}{|l|}{ Male } & 0.67 & 0.64 & 1.60 & 0.59 & 1.66 & 1.97 & 70.72 \\
\hline \multicolumn{3}{|l|}{ Female } & 0.69 & 0.62 & 1.65 & 0.55 & 1.55 & 1.99 & 71.78 \\
\hline \multicolumn{3}{|c|}{$\mathrm{PS} \times \mathrm{SA}$} & NS & NS & NS & NS & NS & NS & NS \\
\hline \multicolumn{3}{|l|}{$\mathrm{PS} \times \mathrm{G}$} & NS & NS & NS & NS & NS & NS & NS \\
\hline \multicolumn{3}{|c|}{$\mathrm{SA} \times \mathrm{G}$} & NS & NS & NS & NS & NS & NS & NS \\
\hline \multicolumn{3}{|c|}{$\mathrm{PS} \times \mathrm{SA} \times \mathrm{G}$} & NS & NS & NS & NS & NS & NS & NS \\
\hline
\end{tabular}

PS: production system G: gender, I: intensive, FR: free range, M: male, F: female, SFA: saturated fatty acids, MUFA: monounsaturated fatty acids, PUFA: polyunsaturated fatty acids, NV: nutritive value, AI: atherogenic index, TI: thrombogenic index, h/H: hypocholesterolaemic/hypercholesterolaemic ratio, DFA: desirable fatty acids, SEM: standard error of the mean, NS: not significant. 
Table 7. Fatty acid index values of thigh meat in partridges

\begin{tabular}{|c|c|c|c|c|c|c|c|c|c|}
\hline \multirow{2}{*}{ PS } & \multirow{2}{*}{ SA } & \multirow{2}{*}{$\mathrm{G}$} & \multicolumn{7}{|c|}{ Fatty acids index } \\
\hline & & & MUFA/SFA & $\begin{array}{l}\text { PUFA/SFA } \\
\end{array}$ & $\mathrm{NV}$ & $\mathrm{AI}$ & TI & $\mathrm{h} / \mathrm{H}$ & DFA \\
\hline \multirow{6}{*}{ I } & \multirow{2}{*}{14} & M & 0.94 & 0.99 & 1.87 & 0.35 & 0.95 & 2.83 & 77.81 \\
\hline & & $\mathrm{F}$ & 1.03 & 0.99 & 1.86 & 0.36 & 0.92 & 2.80 & 77.21 \\
\hline & \multirow{2}{*}{16} & M & 0.81 & 0.80 & 1.82 & 0.42 & 1.15 & 2.47 & 75.38 \\
\hline & & $\mathrm{F}$ & 1.14 & 0.96 & 1.85 & 0.37 & 0.89 & 2.75 & 76.71 \\
\hline & \multirow{2}{*}{18} & M & 0.95 & 0.98 & 1.82 & 0.37 & 0.98 & 2.74 & 76.82 \\
\hline & & $\mathrm{F}$ & 1.07 & 1.03 & 2.02 & 0.34 & 0.88 & 3.01 & 78.42 \\
\hline \multirow{6}{*}{ FR } & \multirow{2}{*}{14} & M & 0.70 & 0.69 & 1.62 & 0.46 & 1.24 & 2.04 & 74.55 \\
\hline & & $\mathrm{F}$ & 0.75 & 0.74 & 1.67 & 0.45 & 1.20 & 2.23 & 74.31 \\
\hline & \multirow{2}{*}{16} & M & 0.97 & 0.82 & 1.88 & 0.39 & 1.03 & 2.57 & 76.18 \\
\hline & & $\mathrm{F}$ & 0.94 & 0.89 & 1.80 & 0.39 & 1.00 & 2.59 & 76.70 \\
\hline & \multirow{2}{*}{18} & M & 1.09 & 0.81 & 1.95 & 0.38 & 0.97 & 2.60 & 76.12 \\
\hline & & $\mathrm{F}$ & 1.04 & 0.76 & 1.92 & 0.39 & 1.01 & 2.54 & 76.35 \\
\hline \multicolumn{3}{|l|}{ SEM } & 0.029 & 0.031 & 0.031 & 0.010 & 0.027 & 0.069 & 0.387 \\
\hline \multicolumn{10}{|c|}{ Effects } \\
\hline \multicolumn{3}{|c|}{ Production system } & NS & * & NS & $*$ & * & $*$ & NS \\
\hline \multirow{2}{*}{\multicolumn{3}{|c|}{$\begin{array}{l}\text { Intensive } \\
\text { Free Range }\end{array}$}} & 0.99 & 0.96 & 1.87 & 0.37 & 0.96 & 2.77 & 77.06 \\
\hline & & & 0.91 & 0.78 & 1.81 & 0.41 & 1.07 & 2.43 & 75.70 \\
\hline \multicolumn{3}{|l|}{ Age } & * & NS & NS & NS & * & NS & NS \\
\hline \multicolumn{3}{|l|}{14} & $0.86^{\mathrm{c}}$ & 0.85 & 1.76 & 0.41 & $1.08^{\mathrm{a}}$ & 2.48 & 75.97 \\
\hline \multicolumn{3}{|l|}{16} & $0.96^{\mathrm{b}}$ & 0.87 & 1.84 & 0.39 & $1.02^{\mathrm{ab}}$ & 2.59 & 76.25 \\
\hline \multicolumn{3}{|l|}{18} & $1.04^{\mathrm{a}}$ & 0.89 & 1.93 & 0.37 & $0.96^{\mathrm{b}}$ & 2.72 & 76.93 \\
\hline \multicolumn{3}{|l|}{ Gender } & * & NS & NS & NS & NS & NS & NS \\
\hline \multicolumn{3}{|l|}{ Male } & 0.91 & 0.85 & 1.82 & 0.40 & 1.05 & 2.54 & 76.14 \\
\hline \multicolumn{3}{|l|}{ Female } & 0.99 & 0.89 & 1.86 & 0.38 & 0.99 & 2.65 & 76.62 \\
\hline \multicolumn{3}{|c|}{$\mathrm{PS} \times \mathrm{SA}$} & * & NS & NS & NS & * & NS & NS \\
\hline \multicolumn{3}{|l|}{$\mathrm{PS} \times \mathrm{G}$} & * & NS & NS & NS & NS & NS & NS \\
\hline \multicolumn{3}{|c|}{$\mathrm{SA} \times \mathrm{G}$} & NS & NS & NS & NS & NS & NS & NS \\
\hline \multicolumn{3}{|c|}{$\mathrm{PS} \times \mathrm{SA} \times \mathrm{G}$} & NS & NS & NS & NS & NS & NS & NS \\
\hline
\end{tabular}

PS: production system, SA: slaughter age, G: gender, I: intensive, FR: free range, M: male, F: female, SFA: saturated fatty acids, MUFA: monounsaturated fatty acids, PUFA: polyunsaturated fatty acids, NV: nutritive value, AI: atherogenic index, TI: thrombogenic index, h/H: hypocholesterolaemic / hypercholesterolaemic ratio, DFA: desirable fatty acids, SEM: standard error of the mean, NS: not significant, $*$ P $<0.05$.

${ }_{a, b, c}$ Differences in superscript letters within columns represent significant differences among the groups.

Vitula et al. (2011) found that the dry matter of Alectoris chukar and grey partridges was $271.93 \mathrm{~g}$ to $282.84 \mathrm{~g}$ in $1 \mathrm{~kg}$ breast meat and $274.75 \mathrm{~g}$ to $321.93 \mathrm{~g}$ in $1 \mathrm{~kg}$ thigh meat. These values were found higher compared to our study results. However, in line with the present study, Juzl et al. (2012) reported that the dry matter and protein percentage in breast meat as well as the fat and ash percentage in thigh meat were higher in partridges reared in the intensive systems when compared with free-range system.

Fatty acid profile of meat in animals reared for meat production has recently attracted interest in terms of meat quality and human health. Similar to most traits of animal production, fatty acid profile is affected by both genetic and environmental factors (De Smet et al., 2004). Organic and free-range systems have increased in parallel to the growth in the number of consumers who prefer animal-friendly or organic products. It is believed that welfare and health standards of animals are improved in these production systems, while the products produced in environmentally friendly conditions at higher quality (Castellini et al., 2002). Therefore, free-range or outdoor production systems are recommended due to their long-term positive impact on animal health, even in the face of some disease risks (Barbosa Filho et al., 2005; Santos et al., 2005).

Fat and fatty acids are considered as unhealthy by consumers in many countries. Most people are wary of the health problems arising from the fat texture and fat content of meat. However, fat and fatty acids are at the center of meat nutrients and contribute to the different meat quality traits in fat and meat tissues (Wood et al., 2008). In addition, the composition of meat and fatty acids is critical due to its effect on meat quality and nutritional value (Oz and Celik, 2015).

Gender was found to affect the stearic acid (C18:0) in thigh meat, which was higher in male animals. The fat content of animals and meat increases with a higher slaughter age, and fatty acid profiles also potentially change (Wood et al., 2008). The growth of fattening animals was found to occur primarily under the skin, followed by intramuscular fat deposition (De Smet et al., 2004). However, production system affects the fatty acid profile in poultry species (Husak et al., 2008). Although percentages of thigh meat fat at different slaughter ages are not statistically significant in our study, it has been determined that this ratio is in an increasing trend with the increase of slaughter age (Table 1).

The percentage of fat in thigh meat was also increased in the intensive system. Similar to our findings, various studies also reported that oleic, linoleic, and palmitic acids were higher in partridge meat (Gulsen et al., 2010; Kwiecien et al., 2015). The SFAs, including stearic (C18:0), myristic (C14:0), and palmitic acids (C16:0), are particularly important for their hypocholesterolaemia properties and are associated with coronary heart diseases. 
The MUFA values should be higher than SFA values to obtain a lower LDL cholesterol level and total/HDL cholesterol ratio (FAO/WHO, 2009). The UFA values have positive effects on human health because they autoxidise more easily than SFAs (Mottram, 1998). Notably, oxidative stability decreases when fatty acids contain high amounts of long-chain PUFAs, which negatively affects the flavor of meat (Lu et al., 2008). Both n-3 and n-6, which play a role in human nutrition, are the precursors of eicosanoids, prostaglandins, leukotrienes, and thromboxane, and are important for the regulation of immunological and cardiac vascular systems (Grashorn, 2007). Omega fatty acids containing the $n-3, n-6$, and n-9 fatty acids take part in strengthening the immune system and protecting the body from coronary heart disease. Deficiency of these fatty acids may lead to various skin diseases, asthma, decline in growth, and various types (e.g., breast and prostate) of cancer (Lewis et al., 2000).

Similarly, Gulsen et al. (2010) determined the SFA and UFA values of breast meat in partridges were 32.77 to $37.25 \%$ and 62.76 to $67.23 \%$, respectively. They also found that the $\sum \mathrm{n}-3$ and $\sum \mathrm{n}-6$ values were 0.59 to $0.90 \%$ and 29.79 to $43.97 \%$, respectively.

According to the studies conducted on broilers, which produce the most frequently consumed poultry meat around the world, the type of production system has different effects on the fatty acid index values. For example, Husak et al. (2008) demonstrated that the chickens reared in the free-range system exhibited higher SFA values compared to intensive system. The MUFA level was higher in the intensive system, whereas the PUFA level was higher in the organic production system. These findings are consistent with our study results. We observed that production system did not affect the total fatty acid composition of breast meat in partridges. However, Castellini et al. (2002) found that the SFA and PUFA values in the breast meat were higher in the organic system, whereas the MUFA values were higher in the intensive system. Similar to our results, Sekeroglu et al. (2009) determined that neither deep litter nor a free-range system affected the saturated or unsaturated fatty acid profile of breast meat in broiler chickens. The pasture in the free-range system in our study was exhausted at the end of the $9^{\text {th }}$ week. For this reason, no change in fatty acid composition due to pasture was determined between the free-range and intensive system at 14,16 and 18 weeks of age.

Adequate and balanced fatty acid intake could decrease the risk of coronary heart diseases, hypertension, diabetes, immunity, and inflammatory diseases. Moreover, the balance of fatty acids is important for meat storage over prolonged periods of time and for flavor because of their oxidation during cooking (Zhou et al., 2012).

Therefore, index values (MUFA/SFA, PUFA/SFA, AI, $\mathrm{TI}$, nutritive value, $\mathrm{h} / \mathrm{H}$, and DFA) are commonly used to evaluate the abdominal fat in terms of nutritive value and health. In general, a low PUFA/SFA, which is used to determine the nutritive value of fatty acids, is considered negative $(<0.4)$ because it could cause an increase in cholesterolaemia (Santos-Silva et al., 2002). Similar to the findings of Kwiecien et al. (2015), the percentages of PUFA/SFA in breast and thigh meat were $>0.4$ in our study.
It is preferred that the AI and TI values be less than 1 (Yakan et al., 2012) and higher AI values may be harmful to human health (Ulbricht and Southgate, 1991). The opposite situation is important for the protective effect of unsaturated fatty acids on cardiac vascular diseases (Manso et al., 2009). The AI values of breast and thigh meat were lower than 1 in our study; however, the TI value was higher than 1 for breast meat and was around 1 for thigh meat ( 0.88 vs 1.24 , respectively). The nutritive value $((\mathrm{C} 18: 0+\mathrm{C} 18: 1) / \mathrm{C} 16: 0)$ is an indicator of health for fat content and constitutes an important part of whole fatty acids. Palmitic acid (C16:0) tends to increase the blood cholesterol level, whereas stearic acid (C18:0) does not affect it and oleic acid (C18:1) tends to decrease it (Caneque et al., 2005). In the present study, the nutritive value of thigh meat was the highest level at 18 weeks of slaughter age. Oleic acid was higher while palmitic acid was lower at 18 weeks of age; and this caused the nutritive value to be higher at 18 weeks, which was a remarkable finding of this study. In parallel with the functional effects of fatty acids, the $\mathrm{h} / \mathrm{H}$ value is used for nutrition determination (Ahmed et al., 2015). The h/H value was higher in the thigh meat of partridges reared in the intensive system. The mean DFA value was found between 66.72 and 76.65 .

In the present study, production system was found to have no significant effect on the nutrient composition in breast meat of partridges, while it had a significant effect only on the erucic acid (C22:1n9) percentage of breast meat. However, these variables were observed to differ in thigh meat. Slaughter age significantly affected only the docosahexaenoic acid (C22:6n3) DHA content of breast meat, while many fatty acids in thigh meat differed by slaughter age. Furthermore, the eicosenoic acid (C20:1) in breast meat as well as the stearic acid (C18:0) and the MUFA/SFA index value in thigh meat differed between male and female partridges.

\section{Conclusion}

In conclusion, it was determined that partridges have a good fatty acid composition. Particularly based on the index values for thigh meat, the crude fat ratio, PUFA, UFA, $\sum \mathrm{n}-6, \mathrm{PUFA} / \mathrm{SFA}$, and $\mathrm{h} / \mathrm{H}$ values increased in the intensive system, whereas the SFA, $\sum \mathrm{n}-3$, AI, and TI increased in the free-range system. Especially, the increase in MUFA and UFA values with age in thigh meat compared to breast indicates that thigh meat is enriched in terms of unsaturated fatty acids. This situation may be related to the decrease in thrombogenic index with age. The higher SFA and AI values determined in the free-range system could be considered a negative outcome for the alternative systems that are preferred by consumers in the context of animal welfare.

\section{References}

Alva VIA, Quintana LJA, Gonzales Rebeles IC, Gonzalez AM. 2015. Effect of photoperiod on the production of chukar partridges (Alectoris chukar). International Journal of Poultry Science, 14: 657-663. doi: 10.3923/ijps.2015.657.663.

Aberle ED, Forrest JC, Gerrard DE, Mills EW, Hedrick HB, Judge MD, Merkel RA. 2001. Structure and composition of animal tissues. Princ. Meat Science, 9-45. 
Ahmed ST, Islam MM, Bostami ABMR, Mun HS, Kim YJ, Yang CJ. 2015. Meat composition, fatty acid profile and oxidative stability of meat from broilers supplemented with pomegranate (Punica granatum L.) by-products. Food Chemistry, 188: 481-488. doi:10.1016/j.foodchem.2015.04. 140.

Barbosa Filho JAD, Silva MAN, Silva IJO, Coelho AAD, Savino VJM. 2005. Behavior and performance of broiler strains reared under semi-intensive system with shaded areas. Brazilian Journal of Poultry Science, 7: 209-213. doi:10.1590/s1516-635x2005000400003.

Berri C. 2000. Variability of sensory and processing qualities of poultry meat. World's Poultry Science Journal, 56: 209-224. doi:10.1079/wps20000016.

Bogosavljevic-Boskovic S, Rakonjac A, Doskovic V, Petrovic MD. 2012. Broiler rearing systems: a review of major fattening results and meat quality traits. World's Poultry Science Journal, 68: 217-228. doi:10.1017/s00439339120 0027x.

Boz MA, Sarica M, Yamak US. 2017. Production traits of artificially and naturally hatched geese in intensive and freerange systems II: slaughter, carcass and meat quality traits. British Poultry Science, 58: 166-176. doi: 10.1080/0007 1668.2016.1261998.

Caneque V, Diaz MT, Alvarez I, Lauzurica S, Perez C, De La Fuente J. 2005. The influences of carcass weight and depot on the fatty acid composition of fats of suckling Manchego lambs. Meat Science, 70: 373-379. doi:10.1016/j. meatsci.2005.02.003.

Castellini C, Mugnai C, Dal Bosco A. 2002. Effect of organic production system on broiler carcass and meat quality. Meat Science, 60: 219-225. doi: 10.1016/S0309-1740(01)00124-3.

De Smet S, Raes K, Demeyer D. 2004. Meat fatty acid composition as affected by fatness and genetic factors: a review. Animal Research, 53: 81-98. doi: 10.1051/ animres:2004003.

FAO/WHO, 2009. Fats and fatty acids in human nutrition. Proceedings of the joint FAO/WHO expert consultation. November 10-14, 2008. Geneva, Switzerland. Annals of Nutrition and Metabolism, 55: 5-300.

Gokalp HY, Kaya M, Tulek Y, Zorba O. 2010. Et ve ürünlerinde kalite kontrolü ve laboratuvar uygulama kılavuzu (in Turkish). Erzurum, TURKEY, Academic Press, 5th ed., 287 pp.

Grashorn MA. 2007. Functionality of poultry meat. Journal of Applied Poultry Research, 16: 99-106. doi:10.1093/japr/16.1.99.

Gulsen N, Umucalilar HD, Kirikci K, Hayirli A, Aktumsek A, Alasahan S. 2010. Sunflower oil supplementation alters meat quality but not performance of growing partridges (Alectoris chukar). Journal of Animal Physiology and Animal Nutrition, 94: 196-203. doi:10.1111/j.1439-0396.2008.00899.x.

Husak RL, Sebranek JG, Bregendahl K. 2008. A survey of commercially available broilers marketed as organic, freerange, and conventional broilers for cooked meat yields, meat composition, and relative value. Poultry Science, 87: 2367-2376. doi:10.3382/ps.2007-00294.

Juzl R, Suchy P, Strakova E, Lucie Rusnikova L, Machacek M, Marada P. 2012. Nutritional value of breast and thigh muscle of chukar partridge (Alectoris chukar) under intensive fattening conditions. Acta Veterinaria Brno, 81: 251-255. doi:10.2754/avb201281030251.

Kwiecien M, Kasperek K, Grela E, Jezewska-Witkowska G. 2015. Effect of caponization on the production performance, slaughter yield and fatty acid profile of muscles of Greenleg Partridge cocks. Journal of Food Science and Technology, 52: 7227-7235. doi 10.1007/s13197-015-1856-6.

Lewis NM, Seburg S, Flanagan NL. 2000. Enriched eggs as a source of N-3 polyunsaturated fatty acids for humans. Partially Funded By The University of Nebraska Agricultural Research Division, (J. Series No 12898).
Lu P, Zhang LY, Yin JD, Everts AKR, Li DF. 2008. Effects of soybean oil and linseed oil on fatty acid compositions of muscle lipds and cooked pork flavour. Meat Science, 80: 910-918. doi:10.1016/j.meatsci.2008.04.010.

Manso T, Bodas R, Castro T, Jimeno V, Mantecon AR. 2009. Animal performance and fatty acid composition of lambs fed with different vegetable oils. Meat Science, 83: 511-516. doi:10.1016/j.meatsci.2009.06.035.

Meluzzi A, Sirri F, Castellini C, Roncarati A, Melloti P, Franchini A. 2009. Influence of genotype and feeding on chemical composition of organic chicken meat. Italian Journal of Animal Science, 8: 766-768. doi:10.4081/ijas.2009.s2.766.

Moro GME, Ariki J, De Souza PA, De Souza HBA, De Moraes VMB, Vargas FC. 2006. Carcass income and chemical composition of the native partridge (Rhynchotus rufescens Tinamiformes) meat. Ciencia Rural, 36: 258-262. doi:10.1590/S0103-84782006000100040.

Mottram DS. 1998. Flavour formation in meat and meat productions: a review. Food Chemistry, 62: 415-424. doi:10.1016/S0308-8146(98)00076-4.

Oz F, Celik T. 2015. Proximate composition, color and nutritional profile of raw and cooked goose meat with different methods. Journal of Food Processing and Preservation, 39: 2442-2454. doi:10.1111/jfpp.12494.

Ozek K, Yazgan O, Bahtiyarca Y. 2003. Effects of dietary protein and energy concentrations on performance and carcass characteristics of Chukar Partridges (Alectoris chukar) raised in captivity. British Poultry Science, 3: 419-426.

Peiretti PG, Meineri G. 2008. Effect on growth performance, carcass characteristics, and the fat and meat fatty acid profile of rabbits fed diets with chia (Salvia hispanica L.) seed supplements. Meat Science, 80: 1116-1121. doi:10.1016/ j.meatsci.2008.05.003.

Santos-Silva J, Bessa RJB, Santos-Silva F. 2002. Effect of genotype, feeding system and slaughter weight on the quality of light lambs, II. Fatty acid composition of meat. Livestock Production Science, 77: 187-194. doi:10.1016/S03016226(02)00059-3.

Santos AL, Sakomura NK, Freitas ER, Fortes CMS, Carrilho ENVM. 2005. Comparison of free-range broiler chicken strains raised in confined or semi-onfined systems. Brazilian Journal of Poultry Science, 7: 85-92. doi:10.1590/S1516635X2005000200004.

Sarica M, Ocak N, Turhan S, Kop C, Yamak US. 2011. Evaluation of meat quality from 3 turkey genotypes reared with or without outdoor access. Poultry Science, 90:13131323. https://doi.org/10.3382/ps.2009-00600.

Sarica M, Boz MA, Yamak US, Ucar A. 2019. Effect of production system and slaughter age on some production traits of guinea fowl: Meat quality and digestive traits. South African Journal of Animal Science, 49: 192-199. doi:10.4314/sajas.v49i1.22.

Satchithanandam S, Fritsche J, Rader JI. 2001. Extension of AOAC official method 996.01 to the analysis of standard reference material (srm) 1846 and infant formulas. Journal of AOAC International, 84: 805-813.

Sekeroglu A, Sarica M, Demir E, Ulutas Z. 2009. Effects of housing systems on growth performance, blood plasma constituents and meat fatty acids in broiler chickens. Pakistan Journal of Biological Sciences, 12: 631-636. doi:10.3923/pjbs.2009.631.636.

Ulbricht TL, Southgate DA. 1991. Coronary heart disease: Seven dietary factors. Lancet, 338: 985-992. doi:10.1016/01406736(91)91846-m.

Vitula F, Suchy P, Strakova E, Karaskova K, Zapletal D, Kroupa L. 2011. Energy value of meat in selected species of feathered game. Acta Veterinaria Brno, 80: 197-202. doi:10.2754/avb201180020197.

Wood JD, Enser M, Fisher AV, Nute GR, Sheard PR, Richardson RI, Hughes SI, Whittington FM. 2008. Fat deposition, fatty acid composition and meat quality: a review. Meat Science, 78: 343-358. doi:10.1016/j.meatsci.2007.07.019. 
Yakan A, Aksu Elmali D, Elmali M, Sahin T, Motor S, Can Y. 2012. Carcass and meat quality characteristics of white and multicolor geese under local breeder conditions. Kafkas Universitesi Veteriner Fakultesi Dergisi, 18: 663-670. doi:10.9775/kvfd.2012.6238.

Yamak US. 2015. Artificial breeding of wild birds in Turkey: Partridge breeding case. Indian Journal of Animal Research, 49: 258-261. doi:10.5958/0976-0555.2015.00054.0.

Yamak US, Sarica M, Boz MA, Ucar A. 2016. The effect of production system (barn and free-range), slaughtering age and gender on carcass traits and meat quality of partridges (Alectoris chukar). British Poultry Science, 57: 185-192. doi:10.1080/00071668.2016.1144920.
Yamak US, Sarica M, Boz MA, Ucar A. 2018. Effect of production system (barn and free-range) and slaughter age on some production traits of guinea fowl. Poultry Science, 97: 47-53. doi:10.3382/ps/pex265.

Yamak US, Sarica M, Boz MA, Ucar A. 2020. Effect of production system and age on the growth performance and carcass traits of pheasants (Phasianus colchicus). Annals of Animal Science, 20: 1-11. doi: 10.2478/aoas-2018-0053.

Zhou LJ, Wu H, Li JT, Wang ZY, Zhang LY. 2012. Determination of fatty acid in broiler breast meat by nearinfrared reflectance spectroscopy. Meat Science, 90: 658-664. doi:10.1016/j.meatsci.2011.10.010. 